\title{
The Development and Application of Electric Sealing Pliers
}

\author{
Li Weijun, Wang Qinghao, Pang Yanjun, Liu Bo, Zhao Xinhua, Hai Tianshu, \\ Zhang Xiaoshu, Wang Yi, Jin Jing, Liang Liguo \\ Fushun Power Supply Company, Liaoning Electric Power Company Limited, State Grid, China \\ sptlove51@126.com
}

Keywords: single phase electric meter of intelligent rate controlling, remote meter reading, electric sealing pliers

\begin{abstract}
According to the national requirements of the Power Grid Corp, Our company will replace the existing single-phase into single phase electric meter of intelligent rate controlling, in order to achieve the function of remote meter reading. Customer service center measurement room needs to undertake the task of verifying the about eight hundred thousand electric meter that makes the calibration personnel in inspection test class need to complete three hundred electric energy meter calibration and six hundred sealing work every day. Because the speed of the sealing speed has a direct impact on the completion of a large number of calibration tasks, we developed the electric sealing pliers to ensure the completion of a large number of calibration tasks.
\end{abstract}

\section{Introduction}

According to the national requirements of the Power Grid Corp, Our company will replace the existing single-phase into single phase electric meter of intelligent rate controlling, in order to achieve the function of remote meter reading. Customer service center measurement room needs to undertake the task of verifying the about eight hundred thousand electric meter, that makes the calibration personnel in inspection test class need to complete three hundred electric energy meter calibration and six hundred sealing work every day in recent five years, in order to ensure the successful completion of a large number of calibration tasks[1-3].

The speed of the sealing speed directly affects the number of electrical energy meter every day, which is related to the completion of the overall workload. We now use manual to seal the electric energy meter as shown in Figure 1, this method is time-consuming and inefficient, and Long time operation will cause security risks for fingers, It is not conducive to the completion of mass calibration work. So we developed a new sealing tool - electric sealing pliers to solve the adverse factors, to ensure the successful completion of a large number of calibration tasks[4,5].

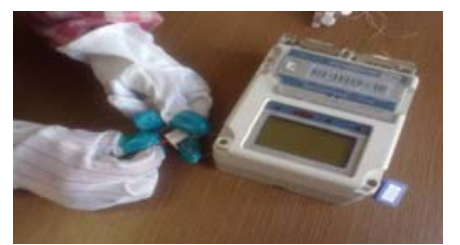

Electric energy meter sealing by manual

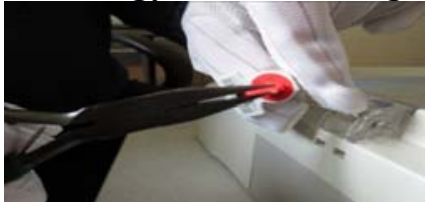

Sealing by flat pliers

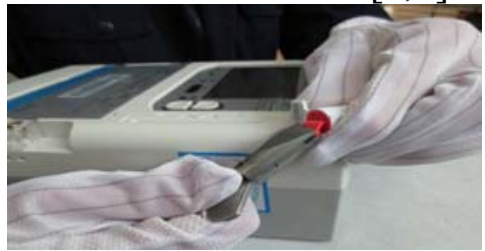

Key sealing by manual

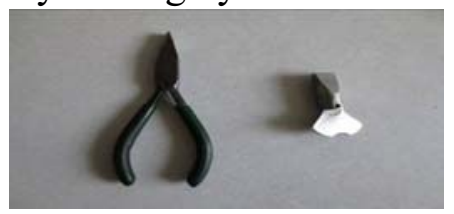

Original sealing tool

Fig. 1 Sealing drawing of manual electric energy meter

\section{The problems of manual electric energy sealing}

We analyze and summarize to the actual use of manual electric energy sealing constantly, the following problems are obtained: 
1) Manual sealing speed is too slow, time-consuming and laborious, low efficiency;

2) Long time manual operation, the operator will fatigue, also easy to cause the error, the index finger and thumb swelling, and even fingers worn phenomenon, there are certain security risks;

3) Improper force will damage the seal by flat pliers;

4) Rotating seal rotating tablet is difficult by key seal sealing;

5) labor intensity of workers is large by Manual.

\section{The development of electric sealing pliers}

According to the problem of electric energy meter sealing by manual, We agreed that the development of a new sealing tools to replace the original manual is the best way to solve the above problems. So we developed the electric sealing pliers, it can be used for a variety of measuring instruments for sealing work. The steps of developing electric sealing pliers are as follows:

(1) We look up the data and company the advantages and disadvantages of the three kinds of power tools in common use as shown in Figure 2, the author design the electric sealing pliers
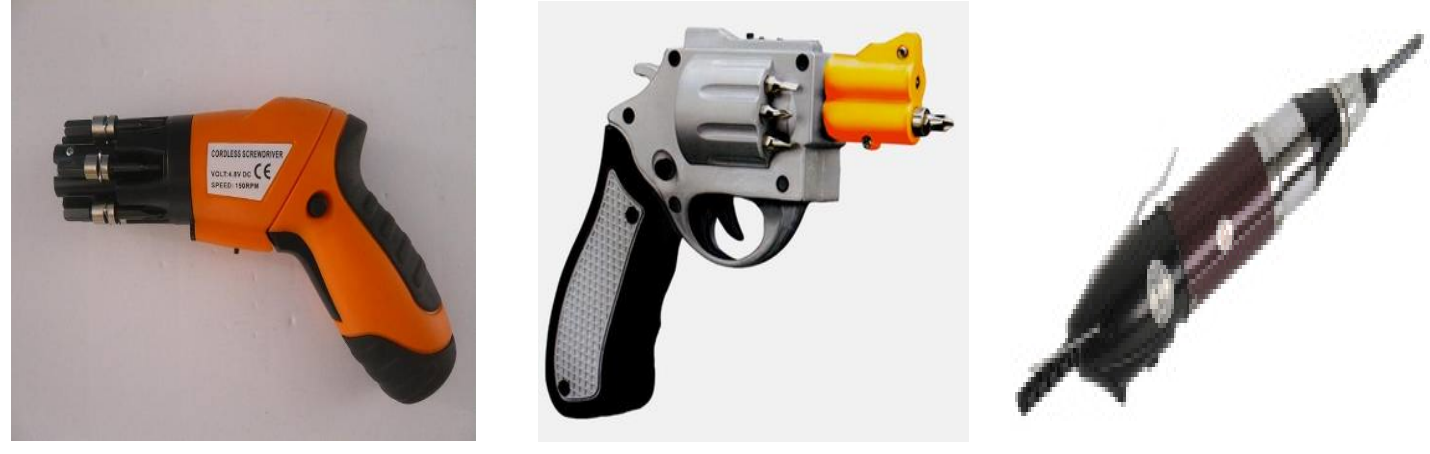

Fig. 2 Three kinds of power tools in common use

The principle of three kinds of power tools is to use the motor as the driving force to drive the output shaft. Their structure is equipped with torque regulation and restriction mechanism for tightening and loosening screw with the tool. The size and weight of the three kinds of electric tools are relatively large, is not conducive to carrying and long time operation. According to the principle and structure of three kinds of electric tools, we design the electric sealing clamp as shown in Figure 3, it can tighten the power rotating sheet seal and easy to break the seal.

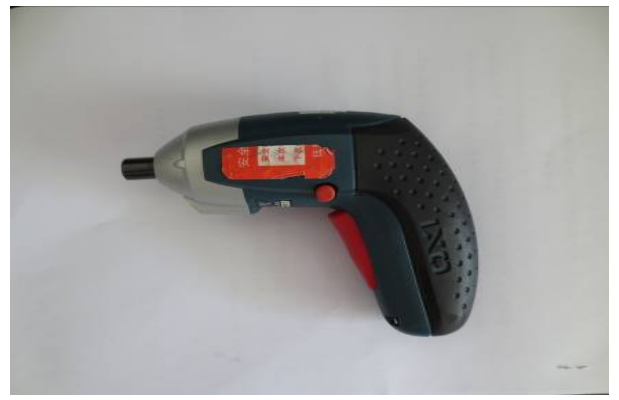

Fig. 3 The electric sealing pliers

(2) The regular shape are the centrifuge rotor of cross-bonding and flat font as shown in Figure 4, according to this we developed the centrifuge rotor of electric sealing pliers.

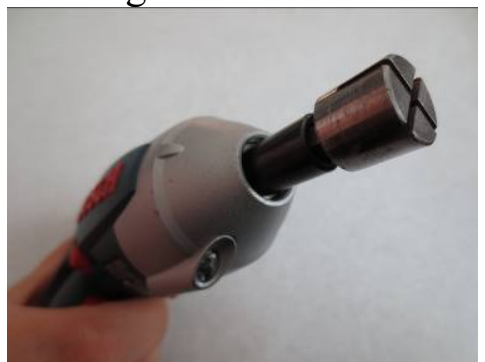

Fig. 4 The centrifuge rotor of cross-bonding 
We use these two kinds of centrifuge rotor to test all kinds of electric energy meter, the conclusion is that the two kinds of centrifuge rotor in insert and tighten the seal is easier, but in breaking the seal rotating piece is very difficult. According to the advantages and disadvantages of the two centrifuge rotors, we developed the centrifuge rotor of the electric sealing pliers as shown in Figure 5. Its torque of the rotating shaft is larger than the cross-bonding type and flat font type, and is easy to break off the rotating plate of the seal

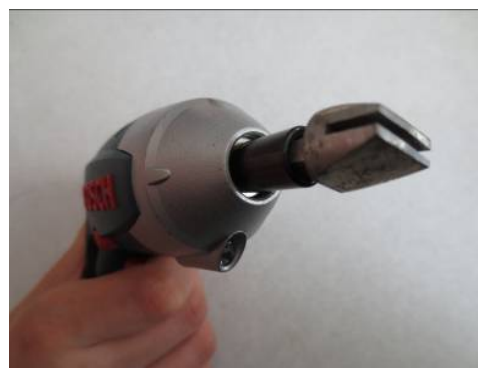

Fig. 5 The centrifuge rotor of electric sealing pliers

\section{The technical principle of electric sealing pliers}

The technical principle of the electric sealing pliers is to supply the energy and the relevant control function for the mechanical part and drive the motor to rotate. The electric sealing clamp is composed of a $220 \mathrm{~V}$ power plug, a charger, a lithium battery, a button, a positive and negative rotation controller, a rotating part and a rotor. Its structure is shown in Fig. 6.

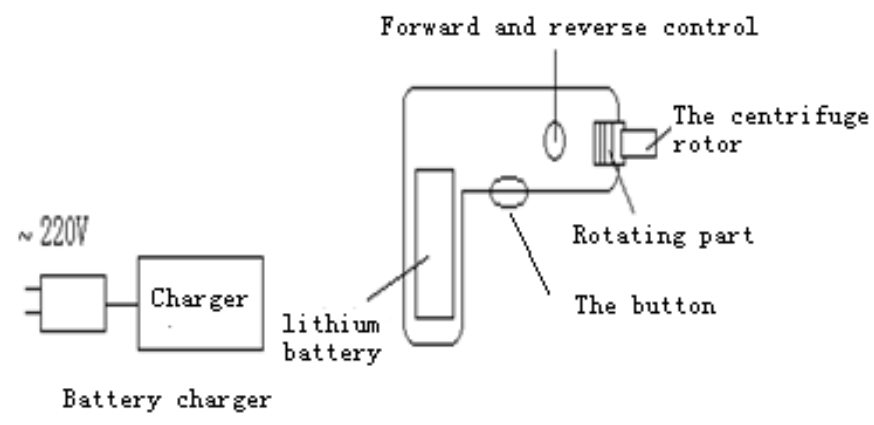

Fig. 6 The structure of electric sealing pliers

The operation of electric sealing pliers is to use the right hand holding the power handle seal clamp, the rotating plate will be hitched by the a centrifuge rotor, press the switch button with the right index finger, turns turn seal rotating plate, screw up the seal, and then breaking the seal rotating plate.

\section{The main parameters of electric sealing pliers}

1) the battery charger is an electronic device used for electric tools, notebook, digital and small portable electronic equipment, It is generally composed of the shell, the power conversion part, the charging detection part, the charging protection part and so on. (1) Charging voltage (V):120-240V/50-60Hz. (2) charging time: 3-5 hours. (3) Rechargeable temperature range (Celsius):5-40.

2) the lithium battery is a kind of two time battery, it depends on the lithium ion moves between the positive pole and the negative pole to work. (1) Voltage (V) 3-6V; (2) service life: 500 times.

3 ) normally open and closed button is switch contact, it is a button with switch on and off

4) the positive and negative controller is to add a set of reversal control contactor to make the two-phase circuit commutation in the main circuit in order to achieve the motor reversal 
5)The speed is set into two files, divided into high speed and low speed, easy to use in the work of choice speed (Rev / min): (1) low speed 0-400r/min, (2) high speed 0-1300r/min.

6) The centrifuge rotor: (1) maximum clamp diameter $(\mathrm{mm}): 10$. (2) the minimum chuck diameter (mm):8. (3) maximum torque: 20N.m.

\section{Application}

(1) Application Situation

In October 2012, the electric sealing pliers were put into the field application; it is used to finish the sealing work of 400 thousand electric energy meters (800 thousand seals). In general, the application effect of electric sealing pliers is satisfactory. It is safe and reliable, simple operation, less error prone, saving time and labor, reduce labor intensity and other advantages have been praised in all aspects. The application of electric seal clamp not only saves cost, but also improves the meter sealing speed, and speed up the completion of the Fushun area of smart meter calibration tasks laid a solid foundation. The effect of using the electric sealing pliers is shown in Figure 7.
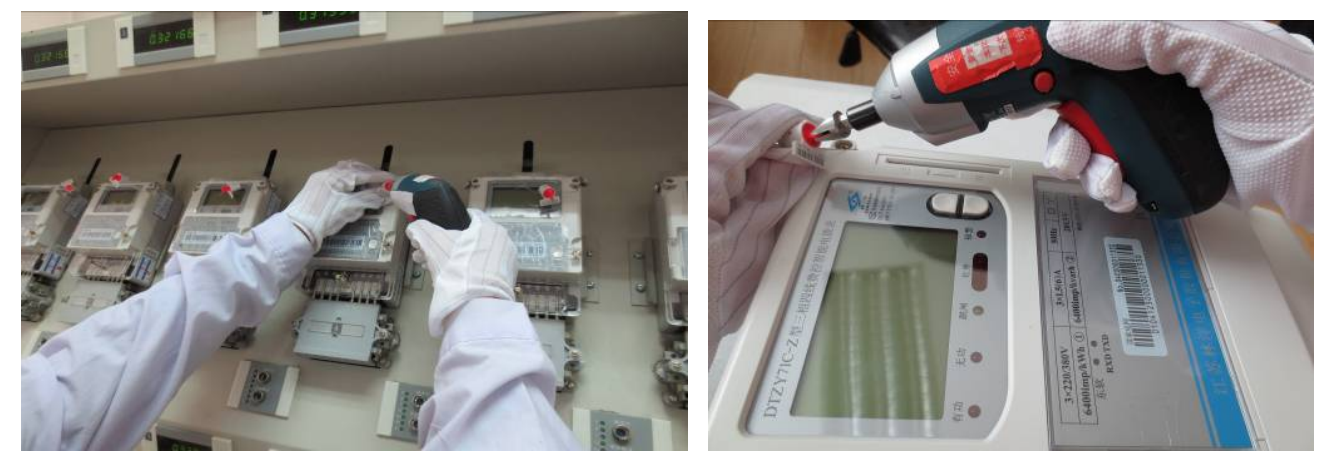

(2) Benefit Analysis

Fig. 7 The effect of electric sealing pliers during use

The efficiency of the electric sealing pliers is quite obvious after being put into use:

1) The electric sealing pliers complete each seal 1 can save time for 15 seconds, the Saving hours of sealing 400 thousand meter ( 800 thousand seals) were as follows: 80 hours saving (10000) $\times 15$ (second $) \div 3600$ (s) $=(3333.3$ hours $)$; Per person per hour is calculated according to the about 40 Yuan, saving hours fee: $3333.3(\mathrm{H})$ x 40 (about 13.3 Yuan) (million Yuan).

2) Due to the original manual sealing method often causing some finger worn, which requires the tape sticking on the fingertip every day. According to a box of tape 10 Yuan, and it needs five boxes per month, so the annual loss is: 10 (Yuan) x 5 (box) $\times 12$ (month) $=600$ (Yuan)

3) The original manual seal is often caused by finger blisters, sore fingers, it will generate an additional part of the medical expenses. Assuming additional medical costs of our team each year is about 400 Yuan. Annual cost savings with electric sealing pliers is : 13.3 (million) 600 (Yuan) 400 (about 13.4 Yuan) (million Yuan).

4) If someone does not pay attention to work, it will puncture and wear fingers. There are security risks, and to make the sealing speed of electric energy meter decreased, impact of workload completion. The newly developed electric sealing pliers realize automatic sealing, avoid the damage of the finger part, eliminate the hidden danger of the safety, and provide the guarantee for the completion of the electric energy meter calibration work.

\section{Conclusion}

1) The electric sealing pliers reduces the labor intensity of the staff;

2) It has small size, light weight, flexible operation, simple and practical;

3) It is safe and reliable, is not easy to make a mistake, do not scratch fingers, eliminating the security risks;

4) It can cost savings of about 130 thousand Yuan per year; 
5) The sealing efficiency is 2 times higher than that of manual operation, and the work efficiency is improved;

6) Its shape is easy to grasp, reduces the fatigue caused by long time grasping, improves the labor productivity.

\section{References}

[1] National Development and Reform Commission, People's Republic of China.DL/T614-2007. People's Republic of China Electric Power Industry Standard - Multifunction Meter Beijing: China Electric Power Press, 2008

[2] Q/GDW354-2009 State Grid Corporation of corporate standards - "Smart Meter Functional Specification" China Electric Power Press, 2009

[3] Q/GDW356-2009 State Grid Corporation of corporate standards - "three-phase smart meter type specification" China Electric Power Press, 2009

[4] State Grid Corporation Q/GDW354-2009. State Grid Corporation of corporate standards smart meter functional specification China Electric Power Press, 2009

[5] Liu Yushun. Measure the success rate of the system acquisition rate improved information collection [J].Volkswagen electricity, 2012(11):12-15 